\title{
An expedient approach to the 2,3,5,6-tetrasubstituted pyridine core of nosiheptide using oxidative cleavage of $2,3,5,8$-tetrasubstituted quinolines
}

\author{
David Taddei, ${ }^{a}$ Cyril Poriel,${ }^{b}$ and Christopher J. Moody ${ }^{a, b, * *}$ \\ ${ }^{a}$ School of Chemistry, University of Nottingham, Nottingham, NG7 2RD, United Kingdom \\ ${ }^{b}$ Department of Chemistry, University of Exeter, Exeter, EX4 4QD, United Kingdom \\ E-mail: c.j.moody@nottingham.ac.uk
}

\begin{abstract}
Pyridine cores 1a and $\mathbf{1 b}$ bearing thiazole rings feature in several natural products such as nosiheptide $\mathbf{2}$ and are often accessed through tedious synthetic routes. We have developed a new approach to the tetrasubstituted pyridine fragment of nosiheptide, which is based on the ozonolysis of tetrasubstituted quinolines, readily obtained by the Meth-Cohn "double Vilsmeier" process.
\end{abstract}

Keywords: Pyridine, quinoline, ozonolysis

\section{Introduction}

The thiopeptide antibiotics ${ }^{1}$ constitute a class of highly modified cyclic peptides with reported biological activity against $\mathrm{MRSA}^{2}$ and malaria, ${ }^{3}$ and which inhibit protein synthesis by interfering with the elongation protein factors in bacteria. ${ }^{4}$ Despite their interesting biological activity, only the total syntheses of micrococcin $\mathrm{P} 1,{ }^{5}$ thiostrepton, ${ }^{6}$ promothiocin $\mathrm{A}^{7}$ and amythiamycin $\mathrm{D}^{8}$ have been reported to date. The total synthesis of nosiheptide $\mathbf{2}$ has not been completed yet, although the preparation of some key fragments has been described in several papers. $^{9-15}$

\footnotetext{
ॠ Chris Moody was Chairman of the RSC Heterocyclic Group during the period 1993-1995.
} 


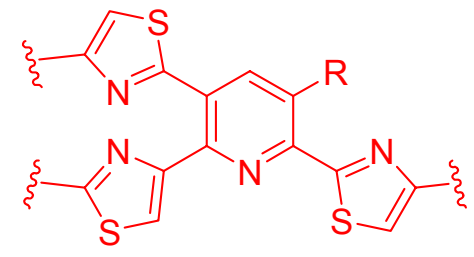

1a $(\mathrm{R}=\mathrm{OH})$

1b $(R=H)$

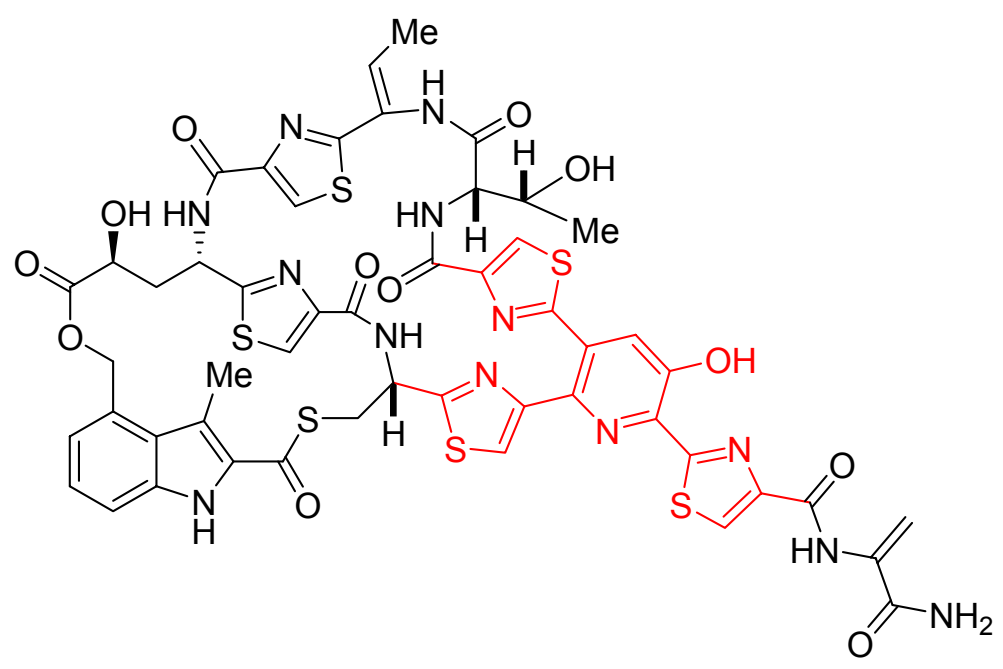

2 nosiheptide

Much of the structural complexity of the thiopeptides is centred on a conserved pyridine core 1a-b, substituted by thiazole rings, and such pyridines have attracted considerable interest from synthetic chemists. Indeed, previous strategies towards this key fragment include the stepwise functionalization of an existing pyridine ring as described by the Shin group, ${ }^{14}$ the cyclization of a suitable 1,5-diketone precursor with ammonia prior to aromatization by dehydrogenation as demonstrated by Ciufolini, ${ }^{16}$ or the sequential regioselective arylation of 2,5,6-tribromopyridine using palladium-catalyzed Negishi and Stille cross-couplings as shown recently by Bach and co-workers. ${ }^{17}$

In contrast, our group has previously focused on a 'biomimetic' hetero-Diels-Alderaromatization sequence under microwave irradiation, ${ }^{18}$ and the Bohlmann-Rahtz reaction between an enamine and an alkynone ${ }^{19}$ to generate the pyridine cores of amythiamycin $\mathrm{D}^{8}$ and promothiocin $\mathrm{A}^{7}$ respectively. Despite these advances there remains a need for a new synthetic approach to access the crucial pyridine core of nosiheptide and related thiopeptide natural products.

\section{Results and Discussion}

Pyridine-5,6-dicarboxylates have recently been prepared by Barré and Perrio by oxidative cleavage of quinolines using ozonolysis or ruthenium tetroxide in biphasic carbon tetrachloride and water. ${ }^{20}$ This was interesting to us since direct precursors to the pyridine core of nosiheptide could be obtained in one key step from readily accessible quinolines. However, the sequential functionalization of these carboxylates into two different thiazoles as in pyridines 1a-b would be difficult to achieve, and this clearly constituted the likely downfall of this methodology.

Therefore, our approach required a pyridine intermediate with two different substituents at the 5- and 6-positions that could be later differentiated into the desired thiazole rings. We 
postulated that the nosiheptide pyridine core 1a could be accessed through classical Hantzsch cyclizations following derivatization of a methyl ester at C-5 and an acetyl moiety at C-6, whilst the third thiazole ring would be introduced through a palladium-catalyzed cross-coupling at C-2 of the 2-chloropyridine intermediate 3 . It was next anticipated that pyridine 4 could be obtained by ozonolysis of the tetrasubstituted quinoline $\mathbf{5}$ and that a Baeyer-Villiger oxidation/hydroxyl protection of quinoline $\mathbf{6}$ would afford the required protected substituent at C-3 (Scheme 1).<smiles>[R]Oc1cc(-c2nc(C)cs2)c(-c2csc(C)n2)nc1-c1nc(C)cs1</smiles><smiles>[R]Oc1cc(C(=O)OC)c(C(C)=O)nc1-c1nc(C)cs1</smiles>
coupling

formation at C-5 and C-6<smiles>[R]Oc1cc(C(=O)OC)c(C(C)=O)nc1Cl</smiles>

$\left.\begin{array}{c}\text { Oxidative } \\ \text { cleavage }\end{array}\right]$<smiles>COc1ccc(C)c2nc(Cl)c(C=O)cc12</smiles><smiles>[R]Oc1cc2c(OC)ccc(C)c2nc1Cl</smiles>

Scheme 1. Retrosynthetic analysis of tetrasubstituted pyridine 1a

Access to the 2-chloroquinoline-3-carbaldehyde 6 was readily accomplished using the Meth-Cohn double-Vilsmeier protocol. ${ }^{21}$ Thus, reacting the commercially available aniline 7 with acetic anhydride in acetonitrile and treating the acetanilide $\mathbf{8}$ under Vilsmeier-Haack conditions with excess phosphorus oxychloride gave the required tetrasubstituted quinoline 6. Oxidation of the 3-formyl moiety of quinoline 6 with $m$-CPBA in dichloromethane was unsatisfactory as the reaction was either incomplete or afforded the quinoline $N$-oxide sideproduct predominantly. However, when an aqueous solution of peracetic acid was slowly added to quinoline $\mathbf{6}$ in chloroform and the intermediate formate ester hydrolyzed with potassium hydrogen carbonate in aqueous methanol, 3-hydroxyquinoline 9 was isolated in $46 \%$ yield over the two steps with recovery of the unreacted starting material. Subsequent protection of the 3hydroxyl group as its isopropyl ether or acetyl ester was performed using standard conditions in 97\% and $93 \%$ yield respectively.

However, when quinolines $\mathbf{1 0 a - b}$ were treated with ruthenium tetroxide in water/chloroform as described by Barré and Perrio, no reaction occurred over $24 \mathrm{~h}$ and the starting materials were recovered. Our attention then turned to the ozonolysis of the same 
substrates below $0{ }^{\circ} \mathrm{C}$. While no product could be isolated when treating the intermediate under oxidative conditions (hydrogen peroxide then sodium hydroxide), we were delighted to find that the desired tetrasubstituted pyridines could be obtained in $39-45 \%$ yield following quenching with dimethyl sulfide and purification of the crude product by flash chromatography on silica gel. The same procedure was also implemented on quinoline 6 and gave the desired product 11c in $45 \%$ yield.

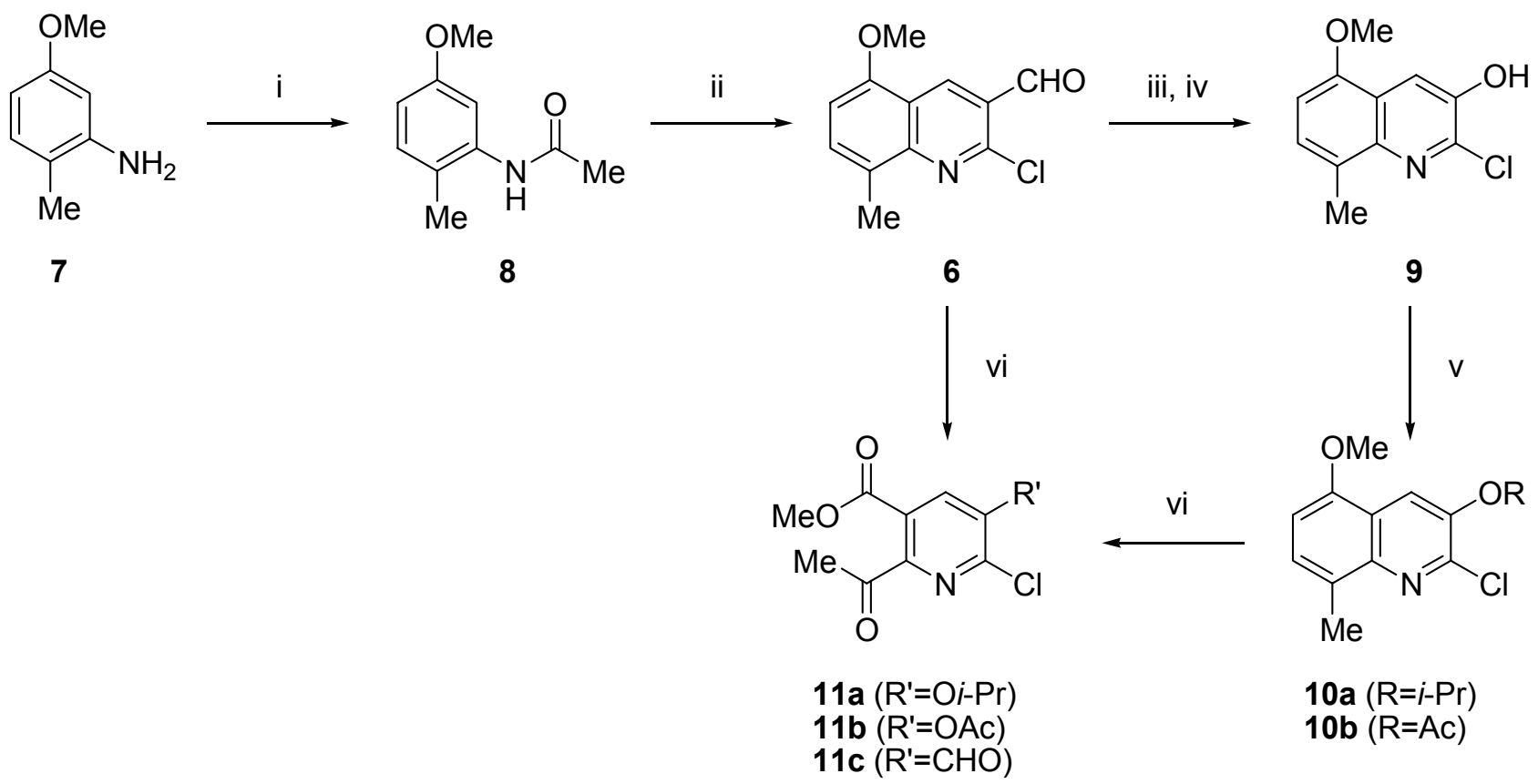

Scheme 2. Reagents and conditions: i) $\mathrm{Ac}_{2} \mathrm{O}, \mathrm{MeCN}, 89 \%$; ii) $\mathrm{POCl}_{3}, \mathrm{DMF}, 0{ }^{\circ} \mathrm{C}, 30$ min then $120{ }^{\circ} \mathrm{C}, 3 \mathrm{~h}, 79 \%$; iii) $\mathrm{MeCO}_{3} \mathrm{H}, \mathrm{CHCl}_{3}, 72 \mathrm{~h}$; iv) $\mathrm{KHCO}_{3}$, aqueous $\mathrm{MeOH}, 46 \%$ (over 2 steps); v) $i$-PrI, $\mathrm{K}_{2} \mathrm{CO}_{3}$, DMF, $75^{\circ} \mathrm{C}, 97 \%\left(\mathrm{R}=i\right.$-Pr, 10a); or $\mathrm{Ac}_{2} \mathrm{O}, \mathrm{K}_{2} \mathrm{CO}_{3}, \mathrm{DMF}, 50{ }^{\circ} \mathrm{C}, 93 \%(\mathrm{R}=\mathrm{Ac}$, 10b); vi) $\mathrm{O}_{3}$, dichloromethane, $-20{ }^{\circ} \mathrm{C}, 30$ min then $\mathrm{Me}_{2} \mathrm{~S},-20{ }^{\circ} \mathrm{C}, 12 \mathrm{~h}, 39-45 \%$.

To conclude, the ozonolysis of 2,3,5,8-tetrasusbtituted quinolines, readily obtained using the Meth-Cohn reaction, gives access to 2,3,5,6-tetrasubstituted pyridines. This approach provides a short and elegant alternative to the traditionally used strategies towards the tetrasubstituted pyridines of thiopeptides. We are currently investigating the palladium-catalyzed cross-coupling of these 2-chloropyridines with various metallated thiazoles.

\section{Experimental Section}

General Procedures. Commercially available reagents were used throughout without further purification unless otherwise stated; solvents were dried by standard procedures. Light petroleum refers to the fraction with bp $40-60{ }^{\circ} \mathrm{C}$. Reactions were routinely carried out under a nitrogen 
atmosphere. Fully characterized compounds were chromatographically homogeneous. IR spectra were recorded in the range $4000-600 \mathrm{~cm}^{-1} .{ }^{1} \mathrm{H}$ and ${ }^{13} \mathrm{C}$ NMR spectra were recorded at $400 \mathrm{MHz}$ and $100 \mathrm{MHz}$ respectively; $J$ values were recorded in $\mathrm{Hz}$. In the ${ }^{13} \mathrm{C}$ NMR spectra, signals corresponding to $\mathrm{CH}, \mathrm{CH}_{2}$, or $\mathrm{CH}_{3}$ groups, as assigned from DEPT, are noted; all others are $\mathrm{C}$.

$\mathrm{N}$-(5-Methoxy-2-methylphenyl)acetamide (8). Acetic anhydride $(3.6 \mathrm{ml}, 32.6 \mathrm{mmol})$ was added dropwise to a stirring solution of 5-methoxy-2-methylaniline 7 (3 g, $21.9 \mathrm{mmol})$ in acetonitrile $(50 \mathrm{ml})$ under a nitrogen atmosphere. The solution was stirred for $12 \mathrm{~h}$, evaporated in vacuo, partitioned between ethyl acetate $(100 \mathrm{ml})$ and saturated aqueous sodium sodium bicarbonate $(200 \mathrm{ml})$. The aqueous layer was further extracted with ethyl acetate and the combined organic extracts were washed with water, dried $\left(\mathrm{MgSO}_{4}\right)$ and evaporated in vacuo. Recrystallization from petroleum ether gave the title compound 8 (3.47 g, 89\%) as colourless needles, mp 95-96 ${ }^{\circ} \mathrm{C}$, (lit., ${ }^{22} \mathrm{mp} 95-95.7^{\circ} \mathrm{C}$ ); $v_{\max }\left(\mathrm{CHCl}_{3}\right) / \mathrm{cm}^{-1} 3452,2938,2837,1688,1618$, $1588,1486,1456,1370,1241,1164,1126,1110,1041,1009,873 ; \delta_{\mathrm{H}}\left(400 \mathrm{MHz} ; \mathrm{CDCl}_{3}\right) 7.43(1$ $\mathrm{H}$, br s, NH), $7.34(1 \mathrm{H}, \mathrm{d}, J$ 2.0, ArH), $7.01(1 \mathrm{H}, \mathrm{d}, J$ 8.2, ArH), $6.60(1 \mathrm{H}, \mathrm{dd}, J 8.2$ and $J$ 2.0, $\mathrm{ArH}), 3.72\left(3 \mathrm{H}, \mathrm{s}, \mathrm{OCH}_{3}\right), 2.12\left(2 \times 3 \mathrm{H}, \mathrm{s}, 2 \times \underline{\mathrm{CH}}_{3}\right) ; \delta_{\mathrm{C}}\left(100 \mathrm{MHz} ; \mathrm{CDCl}_{3}\right) 168.8(\mathrm{C}), 158.2$ (C), $136.5(\mathrm{C}), 130.9(\mathrm{CH}), 121.3(\mathrm{C}), 111.2(\mathrm{CH}), 109.0(\mathrm{CH}), 55.4(\mathrm{Me}), 24.2(\mathrm{Me}), 16.9(\mathrm{Me})$. 2-Chloro-5-methoxy-8-methylquinoline-3-carbaldehyde (6). Phosphorus oxychloride (7 $\mathrm{ml}$, $72 \mathrm{mmol})$ was added dropwise to a stirring solution of the $N$-arylacetamide $8(1.79 \mathrm{~g}, 10 \mathrm{mmol})$ in DMF $(2 \mathrm{ml}, 26 \mathrm{mmol})$ at $0{ }^{\circ} \mathrm{C}$ under a nitrogen atmosphere. The resulting mixture was heated at $130{ }^{\circ} \mathrm{C}$ for $2 \mathrm{~h}$, cooled and poured on to ice-water $(150 \mathrm{ml})$ to precipitate a yellow solid. The mixture was carefully treated with concentrated ammonia to $\mathrm{pH} 9$, extracted with ethyl acetate (3 x $80 \mathrm{ml})$ and the combined organic extracts were dried $\left(\mathrm{K}_{2} \mathrm{CO}_{3}\right)$, evaporated in vacuo and purified by flash chromatography on silica, eluting with ethyl acetate/light petroleum (1:9 then $1: 4)$ to give the title compound $\mathbf{6}(1.86 \mathrm{~g}, 79 \%)$ as fluorescent yellow needles, $\mathrm{mp} 132-133{ }^{\circ} \mathrm{C}$; (Found: $\mathrm{MH}^{+}, 236.0489, \mathrm{C}_{12} \mathrm{H}_{10}{ }^{35} \mathrm{ClNO}_{2}+\mathrm{H}$ requires 236.0478); $v_{\max }\left(\mathrm{CHCl}_{3}\right) / \mathrm{cm}^{-1} 2964,2938$, 2872, 2842, 1692, 1613, 1587, 1575, 1484, 1462, 1438, 1382, 1349, 1322, 1276, 1174, 1151, 1106, 1036, 1004, 977, 951, 854, 823, 636; $\delta_{\mathrm{H}}\left(400 \mathrm{MHz} ; \mathrm{CDCl}_{3}\right) 10.48(1 \mathrm{H}, \mathrm{s}, \mathrm{C} \underline{\mathrm{HO}}), 9.00$ (1 $\mathrm{H}, \mathrm{s}, 4-\mathrm{H}), 7.54(1 \mathrm{H}, \mathrm{d}, J$ 8.0, ArH $), 6.76(1 \mathrm{H}, \mathrm{d}, J$ 8.0, ArH $), 3.98\left(3 \mathrm{H}, \mathrm{s}, \mathrm{OC}_{3}\right), 2.62(3 \mathrm{H}, \mathrm{s}$, $\left.\mathrm{C}_{3}\right) ; \delta_{\mathrm{C}}\left(100 \mathrm{MHz} ; \mathrm{CDCl}_{3}\right) 189.3(\mathrm{CH}), 155.1(\mathrm{C}), 149.6(\mathrm{C}), 148.8(\mathrm{C}), 135.8(\mathrm{C}), 134.0(\mathrm{CH})$, $128.0(\mathrm{CH}), 124.9(\mathrm{CH}), 119.2(\mathrm{C}), 105.4(\mathrm{CH}), 55.9(\mathrm{Me}), 17.1(\mathrm{Me}) ; \mathrm{m} / z(\mathrm{ESI})$ 270/268 $\left(\mathrm{MNa}^{+}, 30 / 100 \%\right), 238 / 236\left(\mathrm{MH}^{+}, 7 / 20 \%\right)$.

2-Chloro-3-hydroxy-5-methoxy-8-methylquinoline (9). Peracetic acid $(8.75 \mathrm{ml}, 40 \%$ in acetic acid/water, $50 \mathrm{mmol})$ in chloroform $(125 \mathrm{ml})$ was added dropwise to a yellow solution of the quinoline-3-carbaldehyde $6(2.5 \mathrm{~g}, 10.6 \mathrm{mmol})$ in chloroform $(125 \mathrm{ml})$ under a nitrogen atmosphere with vigorous stirring. The mixture was stirred for 3 days, extracted with water $(2 \mathrm{x}$ $150 \mathrm{ml})$, and evaporated in vacuo. Methanol/THF (2:1) $(60 \mathrm{ml})$, water $(10 \mathrm{ml})$ and potassium hydrogen carbonate $(2 \mathrm{~g})$ were added sequentially to the yellow residue and the darkening mixture was stirred for $2 \mathrm{~h}$, evaporated in vacuo, and partitioned between ethyl acetate $(100 \mathrm{ml})$ and water $(50 \mathrm{ml})$. The aqueous layer was further extracted with ethyl acetate, dried $\left(\mathrm{MgSO}_{4}\right)$, evaporated in vacuo and purified by flash chromatography on silica, eluting with ethyl 
acetate/light petroleum (4:96 then 15:85) to give the title compound $9(485 \mathrm{mg}, 46 \%)$ as a pale yellow solid, mp 139-140 ${ }^{\circ} \mathrm{C}$; (Found: $\mathrm{MH}^{+}, 224.0476, \mathrm{C}_{11} \mathrm{H}_{10}{ }^{35} \mathrm{ClNO}_{2}+\mathrm{H}$ requires 224.0473); $v_{\max }\left(\mathrm{CHCl}_{3}\right) / \mathrm{cm}^{-1} 3562,2940,2841,1608,1479,1464,1402,1372,1347,1329,1272,1150$, $1100,950,900 ; \delta_{\mathrm{H}}\left(400 \mathrm{MHz} ; \mathrm{CDCl}_{3}\right) 8.01(1 \mathrm{H}, \mathrm{s}, 4-\mathrm{H}), 7.29(1 \mathrm{H}, \mathrm{d}, J$ 8.0, $\mathrm{ArH}), 6.72(1 \mathrm{H}, \mathrm{d}$, $J$ 8.0, $\mathrm{ArH}), 5.75(1 \mathrm{H}, \mathrm{s}, \mathrm{OH}), 3.95\left(3 \mathrm{H}, \mathrm{s}, \mathrm{OC}_{\underline{3}}\right), 2.63\left(3 \mathrm{H}, \mathrm{s}, \underline{\mathrm{C}}_{3}\right) ; \delta_{\mathrm{C}}\left(100 \mathrm{MHz} ; \mathrm{CDCl}_{3}\right)$ 152.7 (C), $144.3(\mathrm{C}), 142.6(\mathrm{C}), 140.2(\mathrm{C}), 127.9(\mathrm{C}), 127.3(\mathrm{CH}), 121.8(\mathrm{C}), 114.7(\mathrm{CH}), 104.9$ (CH), $55.7(\mathrm{Me}), 17.3(\mathrm{Me}) ; \mathrm{m} / z$ (ESI) 226/224 $\left(\mathrm{MH}^{+}, 30 / 100 \%\right)$.

2-Chloro-3-isopropoxy-5-methoxy-8-methylquinoline (10a). 2-Iodopropane $(68 \mu \mathrm{l}, 0.68$ mmol) was added to a stirring solution of the 3-hydroxyquinoline $9(100 \mathrm{mg}, 0.45 \mathrm{mmol})$ and potassium carbonate $(200 \mathrm{mg}, 1.45 \mathrm{mmol})$ at room temperature under a nitrogen atmosphere. The mixture was stirred for $6 \mathrm{~h}$ at $75^{\circ} \mathrm{C}$, cooled, poured on to ice-water $(50 \mathrm{ml})$, and extracted with ethyl acetate $(2 \times 15 \mathrm{ml})$. The combined organic extracts were dried $\left(\mathrm{MgSO}_{4}\right)$, evaporated in vacuo and purified by flash chromatography on silica, eluting with ethyl acetate/light petroleum (2:98) to give the title compound 10a (115 mg, 97\%) as a colourless crystalline solid, mp 140$141{ }^{\circ} \mathrm{C}$; (Found: $\mathrm{MH}^{+}, 266.0959, \mathrm{C}_{14} \mathrm{H}_{16}{ }^{35} \mathrm{ClNO}_{2}+\mathrm{H}$ requires 266.0942 ); $v_{\max }\left(\mathrm{CHCl}_{3}\right) / \mathrm{cm}^{-1}$ 2981, 2938, 1839, 1593, 1478, 1463, 1455, 1368, 1321, 1270, 1153, 1139, 1108, 1048, 980, 954, 884, 844; $\delta_{\mathrm{H}}\left(400 \mathrm{MHz} ; \mathrm{CDCl}_{3}\right) 7.83(1 \mathrm{H}, \mathrm{s}, 4-\mathrm{H}), 7.28(1 \mathrm{H}, \mathrm{d}, J$ 8.0, $\mathrm{ArH}), 6.72(1 \mathrm{H}, \mathrm{d}, J$ 8.0, $\mathrm{ArH}), 4,74\left(1 \mathrm{H}\right.$, sept, $J$ 6.1, $\left.\mathrm{OC}_{\mathrm{HMe}}\right), 3.96\left(3 \mathrm{H}, \mathrm{s}, \mathrm{OC}_{\underline{3}}\right), 2.65\left(3 \mathrm{H}, \mathrm{s}, \underline{\mathrm{C}}_{3}\right), 1.47-1.45(6 \mathrm{H}$, d, $J$ 6.1, $\left.\mathrm{OCH}\left(\mathrm{CH}_{3}\right)_{2}\right) ; \delta_{\mathrm{C}}\left(100 \mathrm{MHz} ; \mathrm{CDCl}_{3}\right) 152.4(\mathrm{C}), 146.7$ (C), 143.7 (C), 141.9 (C), 127.9 (C), $127.0(\mathrm{CH}), 120.8(\mathrm{C}), 112.7(\mathrm{CH}), 104.8(\mathrm{CH}), 72.3(\mathrm{CH}), 55.7(\mathrm{Me}), 21.8(\mathrm{Me}), 17.3$ (Me); $m / z$ (ESI) 268/266 (MH+, 30/100\%).

3-Acetoxy-2-chloro-5-methoxy-8-methylquinoline (10b). Acetic anhydride $(83 \mu \mathrm{l}, 0.88 \mathrm{mmol})$ was added to a solution of the 3-hydroxyquinoline $9(100 \mathrm{mg}, 0.45 \mathrm{mmol})$ and potassium carbonate $(340 \mathrm{mg}, 2.50 \mathrm{mmol})$ in anhydrous DMF at room temperature under a nitrogen atmosphere. The resulting yellowish mixture was stirred at $50{ }^{\circ} \mathrm{C}$ for $6 \mathrm{~h}$, cooled, poured on to ice-water $(15 \mathrm{ml})$ and extracted with ethyl acetate $(2 \times 10 \mathrm{ml})$. The combined organic extracts were dried $\left(\mathrm{MgSO}_{4}\right)$, evaporated in vacuo and purified by flash chromatography on silica, eluting with ethyl acetate/light petroleum (5:95) to give the title compound $\mathbf{1 0 b}$ as a colourless solid (105 mg, 93\%), mp 175-176 ${ }^{\circ} \mathrm{C}$; (Found: $\mathrm{MH}^{+}$, 266.0558, $\mathrm{C}_{13} \mathrm{H}_{12}{ }^{35} \mathrm{ClNO}_{3}+\mathrm{H}$ requires 266.0578); $v_{\max }\left(\mathrm{CHCl}_{3}\right) / \mathrm{cm}^{-1}$ 2960, 2938, 2840, 1774, 1616, 1598, 1463, 1439, 1393, 1359, $1324,1272,1150,1107,1080,1041,1008,951,919,875,824,645 ; \delta_{\mathrm{H}}\left(400 \mathrm{MHz} ; \mathrm{CDCl}_{3}\right) 8.28$ $(1 \mathrm{H}, \mathrm{s}, 4-\mathrm{H}), 7.42(1 \mathrm{H}, \mathrm{d}, J$ 8.0, $\mathrm{ArH}), 6.77\left(1 \mathrm{H}, \mathrm{d}, J\right.$ 8.0, ArH), $3.95\left(3 \mathrm{H}, \mathrm{s}, \mathrm{OC}_{\underline{3}}\right), 2.65(3 \mathrm{H}$, s, $\left.\underline{\mathrm{CH}}_{3}\right), 2.42\left(3 \mathrm{H}, \mathrm{s}, \mathrm{COC}_{3}\right) ; \delta_{\mathrm{C}}\left(100 \mathrm{MHz} ; \mathrm{CDCl}_{3}\right) 168.6(\mathrm{C}), 153.2(\mathrm{C}), 145.3(\mathrm{C}), 144.1(\mathrm{C})$, $139.7(\mathrm{C}), 129.8(\mathrm{CH}), 128.0(\mathrm{CH}), 125.3(\mathrm{CH}), 120.4(\mathrm{C}), 105.2(\mathrm{CH}), 55.7(\mathrm{Me}), 20.7(\mathrm{Me})$, 17.3 (Me); $m / z$ (ESI) 268/266 (MH+ $30 / 100 \%)$.

\section{General procedure for the ozonolysis of quinolines $6,10 \mathrm{a}$ and $10 \mathrm{~b}$}

A stream of ozone in oxygen was bubbled into a slowly stirring solution of the quinoline (1 mmol) in dichloromethane at $-20^{\circ} \mathrm{C}$ for $20-30$ min until the solution was saturated. The gas flow was stopped and stirring was continued for a further $30 \mathrm{~min}$. The solution was flushed with oxygen for $10 \mathrm{~min}$, then nitrogen for $5 \mathrm{~min}$ and treated with dimethyl sulfide $(15 \mathrm{mmol})$ at -20 
${ }^{\circ} \mathrm{C}$, stirred for $12 \mathrm{~h}$ at room temperature, evaporated in vacuo and purified by flash chromatography, eluting with ethyl acetate/light petroleum (2:98 to 10:90) to give the compounds 11a-c.

2-Acetyl-6-chloro-5-isopropoxynicotinic acid methyl ester (11a). (111 mg, 39\%) as a yellow oil; (Found: $\mathrm{MH}^{+}, 272.0689 . \mathrm{C}_{12} \mathrm{H}_{14}{ }^{35} \mathrm{ClNO}_{4}+\mathrm{H}$ requires 272.0690); $v_{\max }\left(\mathrm{CHCl}_{3}\right) / \mathrm{cm}^{-1} 2981$, 2938, 1839, 1593, 1478, 1463, 1455, 1368, 1321, 1270, 1153, 1139, 1108, 1048, 980, 954, 884, 844; $\delta_{\mathrm{H}}\left(400 \mathrm{MHz} ; \mathrm{CDCl}_{3}\right) 7.27(1 \mathrm{H}, \mathrm{s}, 4-\mathrm{H}), 4.67\left(1 \mathrm{H}\right.$, sept, $J$ 6.1, OC$\left.\left(\mathrm{CH}_{3}\right)_{2}\right), 3.91(3 \mathrm{H}, \mathrm{s}$, $\left.\mathrm{OC}_{3}{ }_{3}\right), 2.64\left(3 \mathrm{H}, \mathrm{s}, \mathrm{COC}_{3}\right), 1.41\left(6 \mathrm{H}, \mathrm{d}, J 6.1, \mathrm{CH}\left(\mathrm{C}_{3}\right)_{2}\right) ; \delta_{\mathrm{C}}\left(100 \mathrm{MHz} ; \mathrm{CDCl}_{3}\right) 197.7(\mathrm{C})$, 167.4 (C), 152.2 (C), 143.5 (C), 141.1 (C), 128.8 (C), 119.8 (CH), $73.1(\mathrm{Me}), 53.2(\mathrm{Me}), 26.5$ (Me), 21.7 (Me); $m / z$ (ESI) 274/272 (MH', 30/100\%).

5-Acetoxy-2-acetyl-6-chloronicotinic acid methyl ester (11b). (117 mg, 45\%) as a yellow oil; (Found: $\mathrm{MH}^{+}, 294.0142, \mathrm{C}_{11} \mathrm{H}_{10}{ }^{35} \mathrm{ClNO}_{5}+\mathrm{Na}$ requires 294.0140); $v_{\max }\left(\mathrm{CHCl}_{3}\right) / \mathrm{cm}^{-1} 2955,2399$, $1780,1733,1586,1557,1370,1359,1292,1174,1131,1099,1026,963,857 ; \delta_{\mathrm{H}}(400 \mathrm{MHz}$; $\left.\mathrm{CDCl}_{3}\right) 7.82(1 \mathrm{H}, \mathrm{s}, 4-\mathrm{H}), 3.88\left(3 \mathrm{H}, \mathrm{s}, \mathrm{OC}_{3} 3\right), 2.64\left(3 \mathrm{H}, \mathrm{s}, \mathrm{OCOCH}_{3}\right), 2.38\left(3 \mathrm{H}, \mathrm{s}, \mathrm{COC}_{3} \underline{H}_{3}\right)$; $\delta_{\mathrm{C}}\left(100 \mathrm{MHz} ; \mathrm{CDCl}_{3}\right) 209.9(\mathrm{C}), 166.7(\mathrm{C}), 150.8(\mathrm{C}), 144.6(\mathrm{C}), 143.8(\mathrm{C}), 132.0(\mathrm{CH}), 52.2$ (Me), 26.2 (Me), 19.6 (Me); $m / z$ (ESI) 296/294 ( $\left.\mathrm{MNa}^{+}, 30 / 100 \%\right)$.

2-Acetyl-6-chloro-5-formylnicotinic acid methyl ester (11c). (108 mg, 45\%) as a yellow oil; (Found: $\mathrm{MH}^{+}, 242.0222 . \mathrm{C}_{10} \mathrm{H}_{8}{ }^{35} \mathrm{ClNO}_{4}+\mathrm{H}$ requires 242.0220); $v_{\max }\left(\mathrm{CHCl}_{3}\right) / \mathrm{cm}^{-1} 2956,2881$, $1733,1703,1585,1548,1465,1380,1358,1278,1132,1098,966,914 ; \delta_{\mathrm{H}}\left(400 \mathrm{MHz} ; \mathrm{CDCl}_{3}\right)$ $10.77(1 \mathrm{H}, \mathrm{s}, \mathrm{CHO}), 8.67(1 \mathrm{H}, \mathrm{s}, 4-\mathrm{H}), 3.54\left(3 \mathrm{H}, \mathrm{s}, \mathrm{OC}_{3}\right), 2.15\left(3 \mathrm{H}, \mathrm{s}, \mathrm{COC}_{3} \underline{H}_{3}\right) ; \delta_{\mathrm{C}}(100$ $\mathrm{MHz} ; \mathrm{CDCl}_{3}$ ) 198.2 (C), 187.4 (C), 164.8 (C), 159.4 (C), 154.4 (C), 139.5 (CH), 129,1 (C), 125.4 (C), $53.3(\mathrm{Me}), 27.5$ (Me); $m / z$ (ESI) 244/242 $\left(\mathrm{MH}^{+}, 30 / 100 \%\right)$.

\section{Acknowledgements}

We thank the EPSRC for funding.

\section{References and Footnotes}

1. Bagley, M. C.; Dale, J. W.; Merritt, E. A.; Xiong, X.; Chem. Rev. 2005, 105, 685.

2. Shimanaka, K.; Kinoshita, N.; Iinuma, H.; Hamada, M.; Takeuchi, T.; J. Antibiotics 1994, 47,668 .

3. Clough, B.; Strath, M.; Preiser, P.; Denny, P.; Wilson, R. J. M.; FEBS Lett. 1997, 406, 123.

4. (a) Xing, Y. Y.; Draper, D. E.; Biochemistry 1996, 35, 1581. (b) Heffron, S. E.; Jurnak, F.; Biochemistry 2000, 39, 37.

5. Synthetic efforts have focused on the Bycroft-Gowland proposed structure of micrococcin P1, which has since been revised by Bagley and Merritt. Hence, the NMR spectra of synthetic micrococcin samples have not been found identical to those of the natural product; 
Shin, C.-G.; Okumura, K.; Shigekuni, M.; Nakamura, Y.; Chem. Lett. 1998, 139. Okumura, K.; Nakamura, Y.; Shin, C.; Bull. Chem. Soc. Jpn. 1999, 72, 1561. Okumura, K.; Ito, A.; Yoshioka, D.; Shin, C.-G.; Heterocycles 1998, 48, 1319. Okumura, K.; Suzuki, T.; Nakamura, Y.; Shin, C.-G.; Bull. Chem. Soc. Jpn. 1999, 72, 2483. Ciufolini, M. A.; Shen, Y.-C.; Org. Lett. 1999, 1, 1843. Bagley, M.C.; Merritt, E. A.; J. Antibiot. 2004, 57, 829.

6. (a) Nicolaou, K. C.; Safina, B. S.; Zak, M.; Lee, S. H.; Nevalainen, M.; Bella, M.; Estrada, A. A.; Funke, C.; Zecri, F. J.; Bulat, S.; J. Am. Chem. Soc. 2005, 127, 11159. (b) Nicolaou, K. C.; Zak, M.; Safina, B. F.; Estrada, A. A.; Lee, S. H.; Nevalainen, M.; J. Am. Chem. Soc. 2005, 127, 11176.

7. Bagley, M. C.; Bashford, K. E.; Hesketh, C. L.; Moody, C. J.; J. Am. Chem. Soc. 2000, 122, 3301.

8. Hughes, R. A.; Thompson, S. P.; Alcaraz, L.; Moody, C. J.; J. Am. Chem. Soc. 2005, 127, 15644.

9. Koerber-Plé, K.; Massiot, G.; Synlett 1994, 759.

10. Shin, C.; Nakamura, Y.; Yamada, Y.; Yonezawa, Y.; Umemura, K.; Yoshimura, J.; Bull. Chem. Soc. Jpn. 1995, 68, 3151.

11. Koerber-Plé, K.; Massiot, G. ; J. Heterocycl. Chem. 1995, 32, 1309.

12. Umemura, K.; Tate, T.; Yamaura, M.; Yoshimura, J.; Yonezawa, Y.; Shin, C. ; Synthesis 1995, 1423.

13. Shin, C.; Yamada, Y.; Hayashi, K.; Yonezawa, Y.; Umemura, K.; Tanji, T.; Yoshimura, J.; Heterocycles 1996, 43, 891.

14. Umemura, K.; Noda, H.; Yoshimura, J.; Konn, A.; Yonezawa, Y.; Shin, C. G.; Tetrahedron Lett. 1997, 38, 3539.

15. Umemura, K.; Noda, H.; Yoshimura, J.; Konn, A.; Yonezawa, Y.; Shin, C. G., Bull. Chem. Soc. Jpn. 1998, 71, 1391.

16. Ciufolini, M. A.; Shen, Y. C.; J. Org. Chem. 1997, 62, 3804.

17. Heckmann, G.; Bach, T.; Angew. Chem., Int. Ed. 2005, 44, 1199.

18. Moody, C. J.; Hughes, R. A.; Thompson, S. P.; Alcaraz, L.; Chem. Commun. 2002, 1760.

19. (a) Bohlmann, F.; Rahtz, D.; Chem. Ber. 1957, 90, 2265. (b) Bagley, M. C.; Chapaneri, K.; Dale, J. W.; Xiong, X.; Bower, J.; J. Org. Chem. 2005, 70, 1389. (c) Bagley, M. C.; Dale, J. W.; Ohnesorge, M.; Xiong, X.; Bower, J.; J. Comb. Chem. 2003, 5, 41.

20. Le Bas, M.-D.; Guéret, C; Perrio, C ; Lasne, M.-C. ; Barré, L ; Synthesis 2001, 2495.

21. (a) Meth-Cohn, O.; Narine, B.; Tarnowski, B.; Tetrahedron Lett. 1979, 33, 3111. (b) MethCohn, O.; Narine, B.; Tarnowski, B.; J. Chem. Soc., Perkin Trans. 1 1981, 1520.

22. Akhavan-Tafti, H.; DeSilva, R.; Arghavani, Z.; Eickholt, R. A.; Handley, R. S.; Schoenfelner, B. A.; Sugioka, K.; Sugioka, Y.; Schaapet, A. P.; J. Org. Chem. 1998, 63, 930. 